\title{
Normal Mid-Gestation Fetal Ultrasonography Cannot Reliably Exclude Severe Perinatal Hypophosphatasia
}

\author{
Amish Chinoy ${ }^{a, b} \quad$ Chibuike Iruloh $^{c}$ Bronwyn Kerrd M. Zulf Mughal ${ }^{a}$ b \\ Raja Padidela ${ }^{a, b}$
}

aDepartment of Paediatric Endocrinology, Royal Manchester Children's Hospital, Manchester, UK; baculty of Biology, Medicine \& Health, University of Manchester, Manchester, UK; ' ${ }^{\circ}$ Fetal Medicine Unit, St. Mary's Hospital,

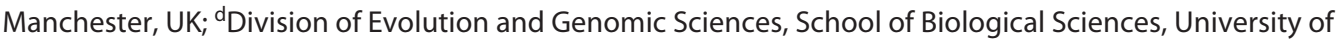
Manchester, Manchester, UK

\section{Established Facts}

- Perinatal hypophosphatasia is the most severe form of hypophosphatasia, characterized by inhibition of bone mineralization due to a mutation in the ALPL gene resulting in deficiency of tissue nonspecific alkaline phosphatase.

- Perinatal hypophosphatasia is usually suggested on antenatal ultrasonography with undermineralization of the long bones, skull, and thoracic cavity - this would usually be performed at mid-gestation.

- Perinatal hypophosphatasia had previously been a lethal condition, although enzyme replacement therapy is now available.

\section{Novel Insights}

- Despite mid-gestation fetal ultrasonography being normal, infants can clearly demonstrate features of perinatal hypophosphatasia later in pregnancy and at birth.

- As mid-gestation fetal ultrasonography cannot be relied upon to exclude perinatal hypophosphatasia, serial antenatal ultrasonography or genetic testing should be considered in families with a family history.

\section{Keywords}

Perinatal hypophosphatasia - Fetal ultrasonography $\cdot A L P L$ gene $\cdot$ Tissue nonspecific alkaline phosphatase

Amish Chinoy and Chibuike Iruloh are joint first authors with equal contributions.

karger@karger.com www.karger.com/hrp

Karger $\stackrel{\text { ' }}{5}$
(C) 2021 The Author(s)

Published by S. Karger AG, Basel

This is an Open Access article licensed under the Creative Commons (http://www.karger.com/Services/OpenAccessLicense), applicable to the online version of the article only. Usage and distribution for commercial purposes requires written permission.

\section{Abstract}

Introduction: Hypophosphatasia is a systemic bone disease characterized by inhibition of bone mineralization due to mutations in the ALPL gene that results in a deficiency of tissue nonspecific alkaline phosphatase. The perinatal form is the most severe. In the past, this form was lethal, although human recombinant enzyme replacement therapy has now
Correspondence to:

Raja Padidela, raja.padidela@ mft.nhs.uk 
been developed and licensed, which improves survival. Perinatal hypophosphatasia is usually suggested on antenatal ultrasonography with undermineralization of the long bones, skull, and thoracic cavity. In the UK, antenatal ultrasonography for fetal anomalies is conducted at mid-gestation (i.e., 18-21 weeks gestational age), and if normal, no further routine scans are performed. Usually, this would identify abnormalities in bone mineralization suggestive of perinatal hypophosphatasia. Cases: We describe 2 cases of perinatal hypophosphatasia where mid-gestation ultrasonography was normal. In the first case, where a previous pregnancy had been terminated for perinatal hypophosphatasia, third trimester ultrasonography revealed skeletal features of hypophosphatasia. In the second case, the diagnosis of perinatal hypophosphatasia was made only immediately after birth. Conclusion: We conclude that serial antenatal ultrasonography or antenatal genetic testing should be considered in all pregnancies with a positive family history of hypophosphatasia, as mid-gestation ultrasonography cannot reliably exclude perinatal hypophosphatasia. This is especially important given that effective enzyme replacement therapy is now available.

(c) 2021 The Author(s).

Published by S. Karger AG, Basel

\section{Introduction}

Hypophosphatasia (HPP) is a systemic bone disease caused by deficiency of tissue nonspecific alkaline phosphatase due to inactivating mutations in the ALPL gene [1]. Tissue nonspecific alkaline phosphatase deficiency results in accumulation of inorganic pyrophosphate, which inhibits bone mineralization [2]. HPP is classified according to age of presentation, with the perinatal form (inherited in an autosomal recessive manner) being the most severe [2]. Perinatal HPP is rare (annual incidence 2-3 per million) [3] and in the past was universally lethal [4]. However, the development of human recombinant enzyme replacement therapy (asfotase alfa) has resulted in an improved survival $[2,5]$.

The possibility of the diagnosis of perinatal HPP is often suggested in utero using antenatal ultrasonography (US), with evidence of shortening, bowing, and angulation of the long bones; deficient or absent ossification of bones (including the skull vault which can be compressed with the US transducer); short, beaded ribs; small or narrow thorax; fractures; polyhydramnios; lung hypoplasia; and pathognomonic mid-diaphyseal osteochondral spurs [6-8]. Most of these features are not specific to HPP, with the sensitivity and specificity of these features in accurately suggesting HPP having never been explored. Differentials for some of these antenatal US findings include osteogenesis imperfecta, campomelic dysplasia, achondrogenesis/hypochondrogenesis, cleidocranial dysplasia, and thanatophoric dysplasia [8]. Even maternal vitamin D insufficiency has been shown to impact on fetal bone development that can result in rachitic appearances [9]. Within the United Kingdom, the National Institute for Health and Care Excellence recommends that detailed US for such structural anomalies is conducted as a routine at mid-gestation (18-21 weeks gestational age [GA]), with no further US performed unless clinically indicated [10]. Here, we describe 2 cases where mid-gestation US was normal, but the infants were subsequently found to have severe perinatal HPP.

\section{Cases}

Case 1

Nonconsanguineous parents were known to be carriers for a mutation in the ALPL gene. This was identified following a previous pregnancy wherein a 20 -week GA scan revealed features suggestive of perinatal HPP. That pregnancy was terminated, with postmortem radiographs and genetic testing consistent with perinatal HPP.

In the current pregnancy, detailed fetal anatomy 2D US performed at 16 and 20 weeks GA (Samsung UGEO WS80A using SC1-6 probe at FR $33 \mathrm{~Hz}$ ) did not reveal any bone abnormalities (Fig. 1). In view of this, the parents declined antenatal genetic testing and continued with the pregnancy. However, concerns about poor intrauterine growth prompted a further detailed scan at 32 weeks GA. This demonstrated generalized undermineralization of the skeleton, generalized micromelia, rachitic changes, and left tibia and fibula fractures (Fig. 2). Genetic testing on chorionic villus biopsy confirmed HPP \{homozygous mutation in ALPL (c.400_401delinsAC > CA [p.Thr134His])\}. Following counseling and discussion about the availability of asfotase alfa, the couple chose to continue with the pregnancy.

A female infant was born at 38 weeks GA by vaginal delivery following induction of labor. She was born with severe skeletal manifestations of HPP (Fig. 3). She required intubation and ventilation for respiratory failure secondary to hypoplastic lungs. Asfotase alfa was commenced on the day of birth, resulting in progressive improvement in bone mineralization over the following months, with concomitant improvement in respiratory status, muscle strength, and motor development.

\section{Case 2}

A female infant was born to consanguineous parents of Pakistani origin. The antenatal period had been unremarkable, and notably her 20-week GA US had been normal. Following premature rupture of membranes, she was delivered by normal vaginal delivery at 34 weeks GA. She was noted to have respiratory distress, with severe skeletal manifestations of perinatal HPP (Fig. 4). Genetic testing confirmed a homozygous mutation in ALPL (c.1336 G > A 

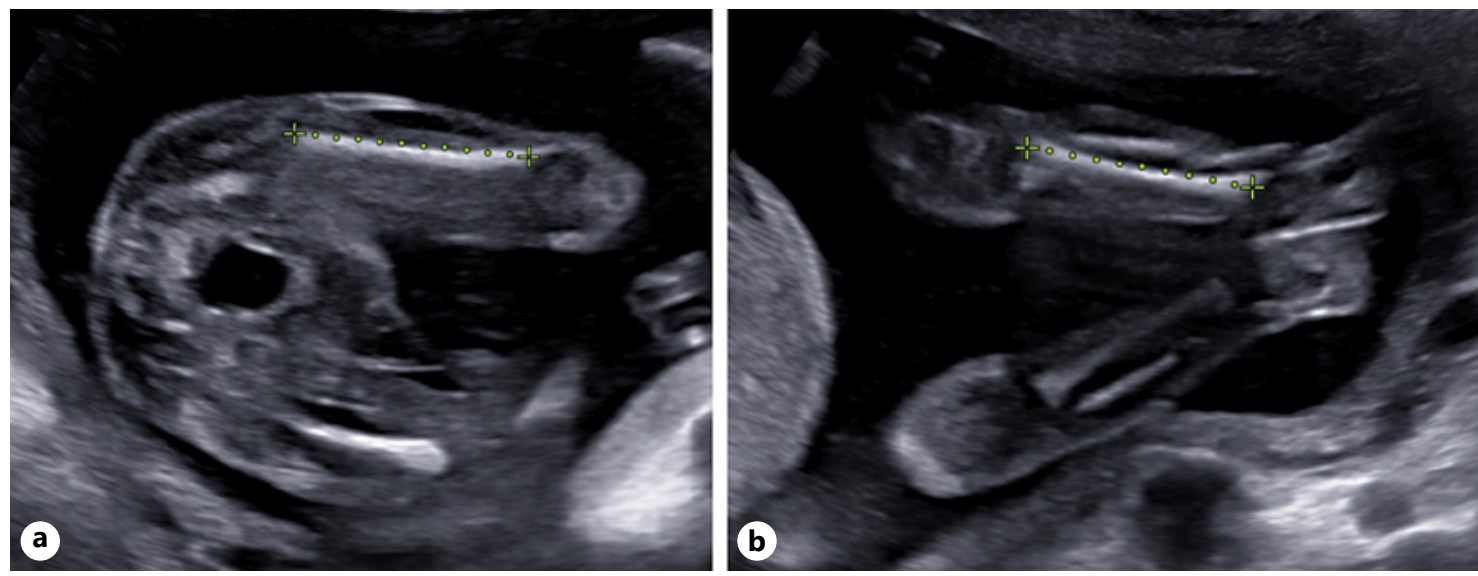

Fig. 1. Detailed fetal anatomy ultrasonography of case 1 performed at 20 weeks gestational age. Normal mineralization and lengths are noted of the femur (a; femoral length $30.52 \mathrm{~mm}$ - equivalent to gestational age of 19 weeks 3 days \pm 13 days) and tibia and fibula (b; tibial length $27.28 \mathrm{~mm}$ - equivalent to gestational age of 19 weeks 6 days \pm 20 days).
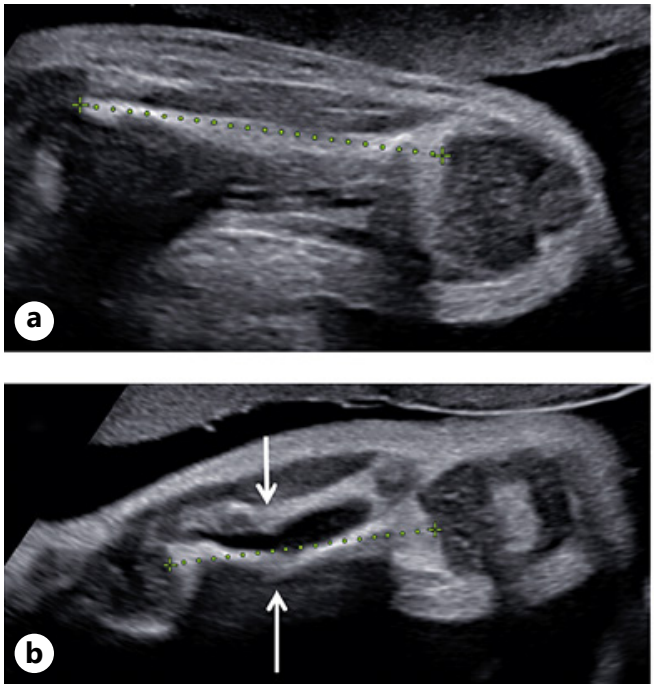

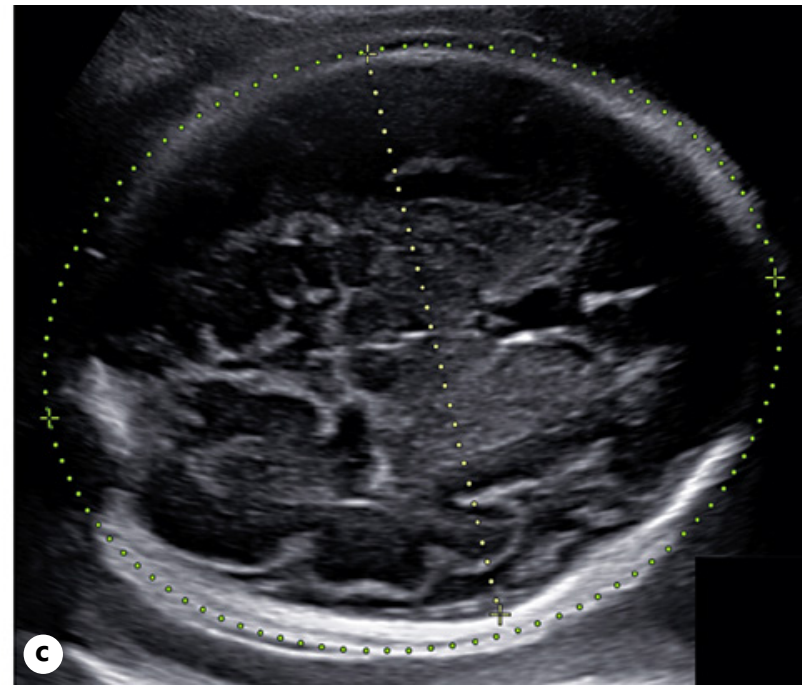

$\mathrm{mm}$ - equivalent to gestational age of 26 weeks 2 days \pm 21 days), undermineralized and fractured (arrows), with splaying of the metaphyseal ends. c Skull vault - noted to be undermineralized, with only islands of ossification, but of normal size.
[p.Ala466Thr]). Asfotase alfa was commenced at 1 month of age, resulting in survival and significant improvement in bone mineralization over the following months.

\section{Discussion}

Since the 1970s, antenatal US has been used as the primary tool for first-line screening of perinatal HPP [11], with genetic confirmation currently undertaken follow-

Fetal Ultrasonography in Perinatal Hypophosphatasia ing suspicious findings on US. The optimal timing of antenatal US for detecting HPP is unclear, with changes evident in some cases as early as 11-14 weeks GA, although most have argued that mid-gestation/second trimester will identify features of perinatal HPP in most instances $[8,12]$. However, the cases of severe perinatal HPP reported above had normal mid-gestation US, with case 1 demonstrating abnormalities typical of perinatal HPP only at 32 weeks GA instead, even though a previously 

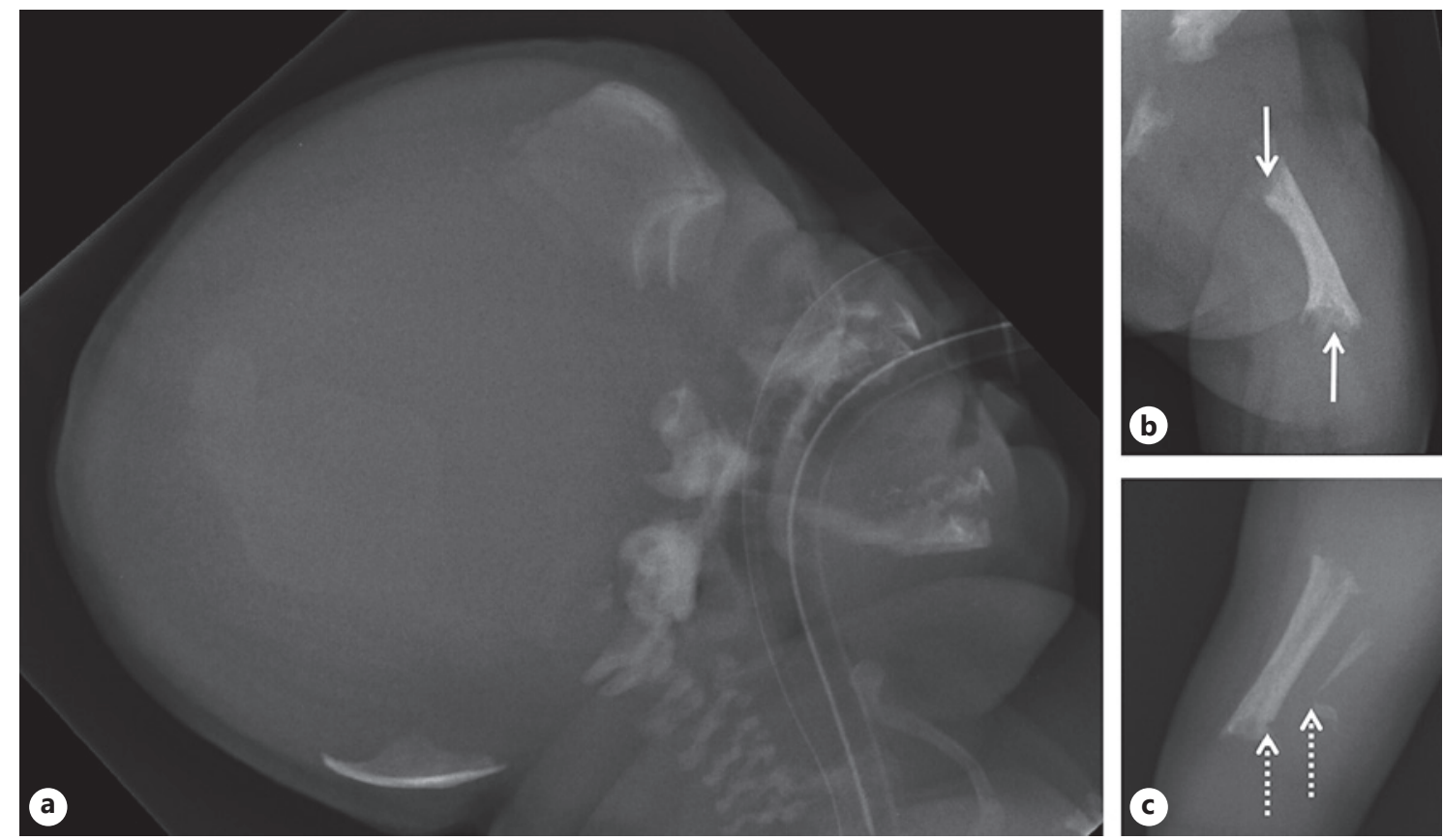

Fig. 3. Plain radiographs of case 1 performed at birth. a Very poorly ossified skull, with almost the entire skull vault not visible due to undermineralization, and only an "island" of ossification visible. b Left femur noted to be extremely small and bowed, with rachitic appearances at the metaphyses (solid white arrows). c Fractured left tibia and fibula (dotted white arrows), with very poor ossification of the fibula.
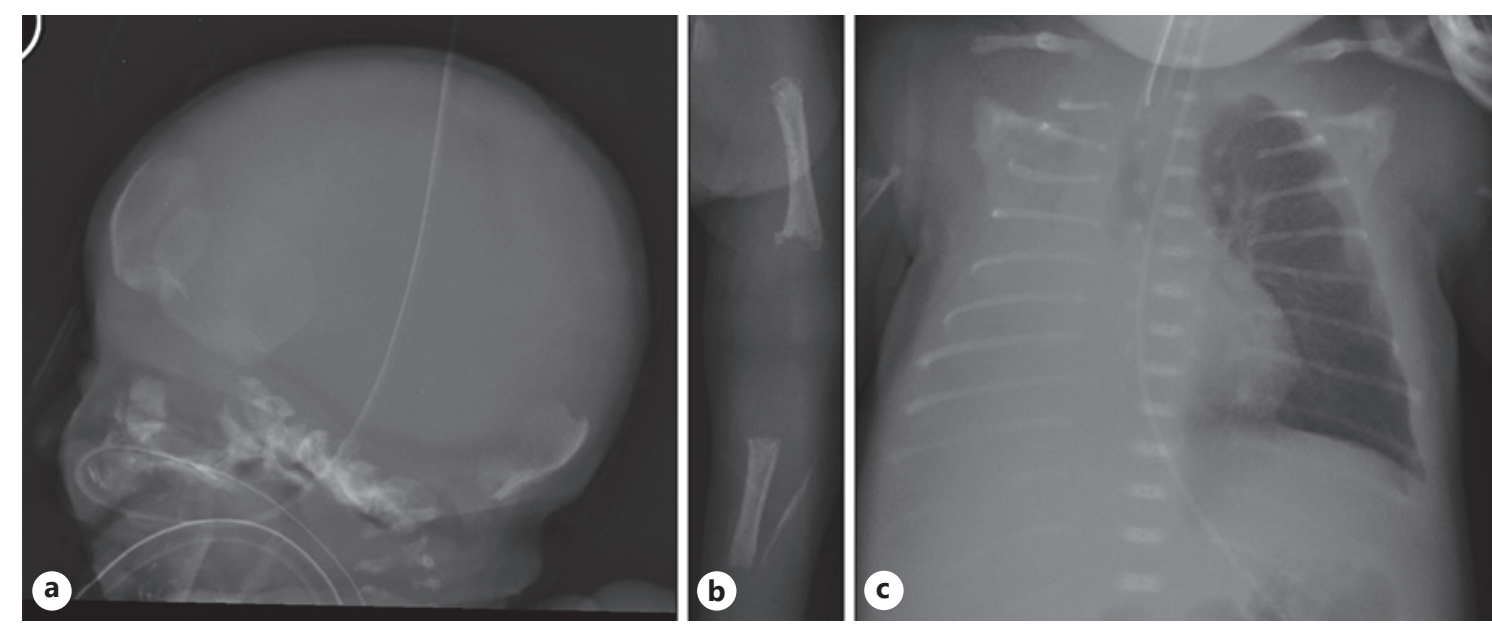

Fig. 4. Plain radiographs of case 2 performed at birth. Similar to case 1 , a very poorly ossified skull is noted with islands of ossified bone visible only (a); left lower limb demonstrating undermineralized, poorly ossified, short, rachitic bones (b). A chest X-ray (c) highlights the slender, poorly ossified ribs, which, together with the muscle weakness seen in hypophosphatasia, contributes to the severe respiratory compromise for these infants due to inadequacy of chest wall movement. In addition, lung hypoplasia is also observed which further contributes to the respiratory issues. This X-ray also highlights further respiratory issues for this infant from right lung collapse. 
affected sibling had had scan abnormalities at 20 weeks GA.

It is unclear why the cases of perinatal HPP we have described had normal mid-gestation US but severe manifestations during the third trimester and at birth. Although early case reports had suggested that manifestations of HPP on the second trimester US indicated severity of disease, Wenkert et al. [13] demonstrated that infants with a milder form of HPP (benign perinatal) also demonstrate US changes during the second trimester that may improve during the third trimester. These cases of benign perinatal HPP are also suggested by normal fetal chest and abdominal size, fetal crowding, absence of fractures in utero, and femoral shortening that is more within the normal range. The majority were born to mothers with a heterozygous mutation in the $A L P L$ gene. In our cases, we postulate that it is the cumulative fetal accumulation of inorganic pyrophosphate during pregnancy that may have resulted in progressive skeletal demineralization which only became apparent on imaging later in pregnancy.

In most pregnancies, serial antenatal US beyond the mid-gestation scan is impractical, given the rarity of perinatal HPP. However, in the presence of positive family history, as in case 1, we would suggest serial antenatal US beyond mid-gestation as a routine to identify those instances where the mid-gestation scan was normal. It must also be recognized that antenatal US is highly operator dependent [14]. Therefore, one could argue that in such cases with a positive family history, US should be undertaken by an experienced sonographer. With evolution of genetic testing, it could also be argued that in such cases, antenatal genetic testing should be offered as routine even with normal mid-gestation US $[15,16]$. Timely diagnosis is not only important for parental counseling but also to make postnatal arrangements given that effective enzyme replacement therapy is available.
To conclude, we have reported 2 cases of severe perinatal HPP where the mid-gestation US was normal. Therefore, we propose that standard mid-gestation US cannot be relied upon to exclude a diagnosis of perinatal HPP. The presence of a positive family history should serve as an important clinical indication, for which we would recommend routine antenatal genetic testing be offered to diagnose perinatal HPP, as well as US later into pregnancy.

\section{Statement of Ethics}

The study is exempt from ethics committee approval as it simply describes cases retrospectively. However, written informed consent was obtained from the parents for publication of this case report and any accompanying images.

\section{Conflict of Interest Statement}

The authors have no conflicts of interest to declare.

\section{Funding Sources}

As this was a retrospective review of clinical cases, no funding was required or necessary for the preparation of the manuscript.

\section{Author Contributions}

A.C. contributed to conception, drafting, and revising; C.I., B.K., M.Z.M., and R.P. contributed to conception and revising.

\section{Data Availability Statement}

No data have been generated or analyzed in this case report.

\section{References}

1 Weiss MJ, Cole DE, Ray K, Whyte MP, Lafferty MA, Mulivor RA, et al. A missense mutation in the human liver/bone/kidney alkaline phosphatase gene causing a lethal form of hypophosphatasia. Proc Natl Acad Sci USA. 1988;85(20):7666-9.

2 Whyte MP. Hypophosphatasia: an overview for 2017. Bone. 2017;102:15-25.

3 Taketani T, Onigata K, Kobayashi H, Mushimoto Y, Fukuda S, Yamaguchi S. Clinical and genetic aspects of hypophosphatasia in Japanese patients. Arch Dis Child. 2014;99(3):211-5.
4 Leung EC, Mhanni AA, Reed M, Whyte MP, Landy H, Greenberg CR. Outcome of perinatal hypophosphatasia in manitoba mennonites: a retrospective cohort analysis. JIMD Rep. 2013;11:73-8.

5 Whyte MP, Greenberg CR, Salman NJ, Bober MB, McAlister WH, Wenkert D, et al. Enzyme-replacement therapy in life-threatening hypophosphatasia. N Engl J Med. 2012; 366(10):904-13.
6 Guguloth A, Aswani Y, Anandpara KM. Prenatal diagnosis of hypophosphatasia congenita using ultrasonography. Ultrasonography. 2016;35(1):83-6.

7 Linglart A, Salles JP. Hypophosphatasia: the contribution of imaging. Arch Pediatr. 2017; 24(5S2):5S74-5S9.

8 Offiah AC, Vockley J, Munns CF, Murotsuki J. Differential diagnosis of perinatal hypophosphatasia: radiologic perspectives. Pediatr Radiol. 2019;49(1):3-22. 
9 Mahon P, Harvey N, Crozier S, Inskip H, Robinson S, Arden N, et al. Low maternal vitamin D status and fetal bone development: cohort study. J Bone Miner Res. 2010;25(1):14-9.

10 NICE Clinical Guidelines. Antenatal care for uncomplicated pregnancies (clinical guideline CG62). London: National Institute for Health and Care Excellence; 2008.

11 Rudd NL, Miskin M, Hoar DI, Benzie R, Doran TA. Prenatal diagnosis of hypophosphatasia. N Engl J Med. 1976;295(3):146-8.
12 Tongsong T, Pongsatha S. Early prenatal sonographic diagnosis of congenital hypophosphatasia. Ultrasound Obstet Gynecol. 2000; 15(3):252-5.

13 Wenkert D, McAlister WH, Coburn SP, Zerega JA, Ryan LM, Ericson KL, et al. Hypophosphatasia: nonlethal disease despite skeletal presentation in utero (17 new cases and literature review). J Bone Miner Res. 2011; 26(10):2389-98.

14 Tolsgaard MG, Todsen T, Sorensen JL, Ringsted C, Lorentzen T, Ottesen B, et al. International multispecialty consensus on how to evaluate ultrasound competence: a Delphi consensus survey. PLoS One. 2013;8(2): e57687.
15 Henthorn PS, Whyte MP. Infantile hypophosphatasia: successful prenatal assessment by testing for tissue-non-specific alkaline phosphatase isoenzyme gene mutations. Prenat Diagn. 1995;15(11):1001-6.

16 Taillandier A, Domingues C, De Cazanove C, Porquet-Bordes V, Monnot S, Kiffer-Moreira $\mathrm{T}$, et al. Molecular diagnosis of hypophosphatasia and differential diagnosis by targeted next generation sequencing. Mol Genet Metab. 2015;116(3):215-20. 